\title{
Early postoperative treatment of mastectomy scars using a fractional carbon dioxide laser: a randomized, controlled, split-scar, blinded study
}

\author{
Hyun Woo Shin, Sangwoo Suk, Seoung Wan Chae, Kun Chul Yoon, Junekyu Kim \\ Department of Plastic and Reconstructive Surgery, Kangbuk Samsung Hospital, Sungkyunkwan University School of Medicine, Seoul, Korea
}

Background Mastectomy leaves unsightly scarring, which can be distressing to patients. Laser therapy for scar prevention has been consistently emphasized in recent studies showing that several types of lasers, including fractional ablation lasers, are effective for reducing scar formation. Nonetheless, there are few studies evaluating the therapeutic efficacy of ablative $\mathrm{CO}_{2}$ fractional lasers (ACFLs).

Methods This study had a randomized, comparative, prospective, split-scar design with blinded evaluation of mastectomy scars. Fifteen patients with mastectomy scars were treated using an ACFL. Half of each scar was randomized to "A," while the other side was allocated to group "B." Laser treatment was conducted randomly. Scars were assessed using digital photographs of the scar and Vancouver scar scale (VSS) scores. Histological assessments were also done.

Results The mean VSS scores were $2.20 \pm 1.28$ for the treatment side and $2.96 \pm 1.40$ for the control side. There was a significant difference in the VSS score between the treatment side and the control side $(P=0.002)$. The mean visual analog scale (VAS) scores were $4.13 \pm 1.36$ for the treatment side and $4.67 \pm 1.53$ for the control side. There was a significant difference in VAS score between the treatment side and the control side $(\mathrm{P}=0.02)$.

Conclusions This study demonstrated that early scar treatment using an ACFL significantly improved the clinical results of the treatment compared to the untreated scar, and this difference was associated with patient satisfaction.

Keywords Laser therapy / Cicatrix / Mastectomy
Correspondence: Hyun Woo Shin Department of Plastic and Reconstructive Surgery, Kangbuk Samsung Hospital, Sungkyunkwan University School of Medicine, 29 Saemunan-ro, Jongno-gu, Seoul 03181, Korea

Tel: +82-2-2001-2178

Fax: +82-2-2001-2177

E-mail:shw.shin@samsung.com

Received: December 18, 2020 • Revised: April 16, 2021 • Accepted: May 27, 2021

pISSN: 2234-6163 • elSSN: 2234-6171 • https://doi.org/10.5999/aps.2020.02495・Arch Plast Surg 2021;48:347-352

\section{INTRODUCTION}

Breast cancer is the most widespread type of cancer among women, affecting 2.1 million women each year and causing the most cancer-related deaths among women [1]. It has been estimated that 627,000 women died from breast cancer in 2018, accounting for about $15 \%$ of all cancer deaths in women [2]. The incidence of breast cancer is steadily increasing, with 22,550 new cases and 2,353 deaths reported in 2015, based on statistics from the Korea Central Cancer Registry [3]. Since the 5-year survival rate of women with breast cancer is relatively high, at $89 \%$ [1], breast reconstruction has become a crucially important part of the care plan for these patients, and the demand for post-mastectomy breast reconstruction is simultaneously in- 
creasing [4]. However, mastectomy leaves unsightly scarring, and cosmetically unappealing scars cause distress in patients [5]. In addition, there is growing concern about scarring and patients are increasingly focusing on cosmetic outcomes. Thus, the proper treatment of breast reconstruction scars is important, and many procedures have been used to reduce scar formation.

Laser treatment has been consistently emphasized in recent studies for scar prevention, showing that several types of lasers, including fractional ablation lasers, are effective for reducing scar formation [6-9]. Ablative $\mathrm{CO}_{2}$ fractional lasers (ACFLs) heat and vaporize superficial skin layers. Then, the healing process induces new collagen formation and collagen remodeling, which are responsible for scar improvement [9]. Nevertheless, few studies have evaluated the therapeutic effect of ACFLs. Moreover, recent research has begun to focus on early scar interventions. Therefore, the purpose of this study was to evaluate the efficacy of ACFL treatment for breast reconstruction scarring in the early postoperative period.

\section{METHODS}

\section{Study design and patients}

This study had a randomized, comparative, prospective, splitscar design with blinded evaluation of mastectomy scars. Between April 2019 and November 2019, 15 patients who underwent total mastectomy and immediate, implant-based breast reconstruction were treated using an ACFL (10,600 nm carbon dioxide; Lutronic Corp., Goyang, Korea) Mastectomy was performed using the same method in all patients. The incision was made from the lateral aspect to the nipple in a linear shape, and closure was based on subcutaneous buried Vicryl sutures and nylon sutures for the skin. Treatment was initiated after suture removal. Half of the scar was randomized to "A," while the other side was allocated to "B." Laser treatment was conducted randomly (Fig. 1). The treatment parameters ranged from 22 to 38 $\mathrm{mJ}$, at a density of 300 spots $/ \mathrm{cm}^{2}$ in the static operating mode, mainly starting at the time of suture removal. Only one pass was made using a scan area of $4 \times 4 \mathrm{~mm}$ in the static mode, and no other treatment (e.g., tension-relieving devices or silicone gels) was administered. After laser treatment, the patients were advised to apply hydrocolloid dressing (Duoderm; ConvaTec, Oklahoma City, OK, USA) for a 1-week. All patients provided written informed consent before participating in the trial.

\section{Scar assessment}

With the same background, exposure, and light source, and using the same digital camera (750D; Canon, Tokyo, Japan), photographs of scars were taken both before and 6 months after la-

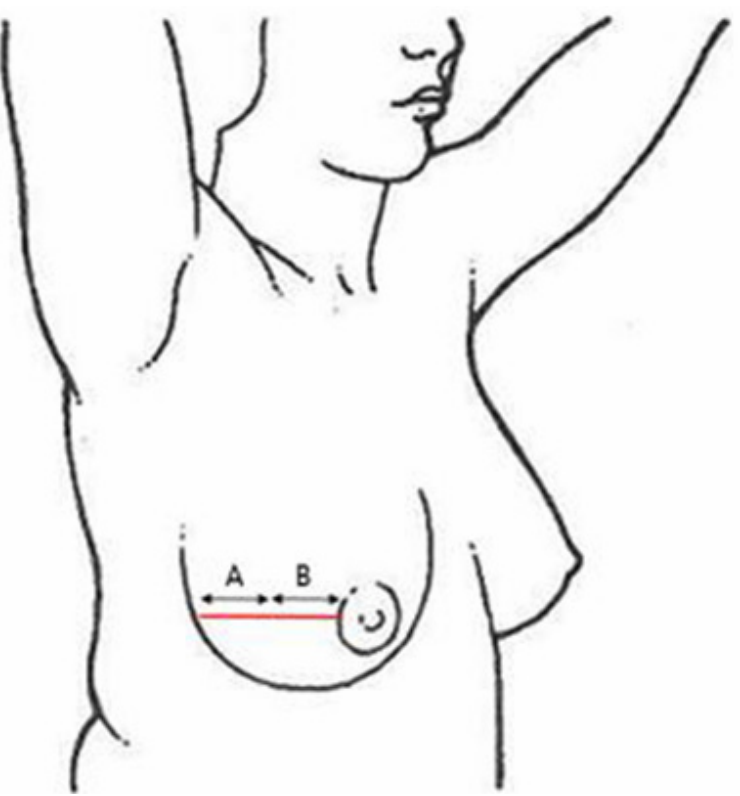

Fig. 1. Study design of the mastectomy scars. Half of each scar was randomized to "A," while the other side was allocated to "B." Laser treatment was conducted randomly. The red line is the mastectomy scar.

ser treatment. Three blinded physicians independently graded the treatment outcomes using the Vancouver scar scale (VSS), which evaluates scar vascularity, pigmentation, and height from 0 to 3 and pliability from 0 to 5; a score of 0 indicates similarity to normal skin, while the maximum score represents the worst possible scar. Patient satisfaction was evaluated by overall scar appearance using a visual analog scale (VAS), ranging from 0 to 10 , where a score of 0 indicates similarity to normal skin and 10 represents the worst possible scar. To investigate histological changes after ACFL treatment, patients who underwent tissue expander insertion were asked to undergo biopsies from the scar area. Three patients agreed, with a total of six biopsies at the time of implant change surgery, and three from ACFL-treated scars and three from untreated control scars. The biopsied tissues were formalin-fixed and paraffin-embedded. For routine tissue pathological evaluation, a section of 5- $\mu \mathrm{m}$ thickness was cut and stained with hematoxylin and eosin. Masson's trichrome stain was used to visualize collagen fibers.

\section{Statistical analyses}

Changes in VSS and VAS scores between the treatment and control halves of scars were compared using the paired t-test. A $\mathrm{P}$-value $<0.05$ was considered to indicate statistical significance. All analyses were performed with SPSS for Windows, version 20.0 (IBM Corp., Armonk, NY, USA). 


\section{RESULTS}

Fifteen patients with 15 scars completed the treatment protocol and follow-up. Table 1 shows the demographic and clinical characteristics of the study group. The average age was 43.4 years. The average length of the scar was $8.3 \mathrm{~cm}$, resulting in an average treatment length of $4.2 \mathrm{~cm}$.

ACFL treatment improved clinical outcomes (Figs. 2, 3). The mean VSS scores were $2.20 \pm 1.28$ for the treatment side and

Table 1. Demographic and clinical characteristics of the study group

\begin{tabular}{lc}
\hline Characteristic & $\begin{array}{c}\text { Study population } \\
(\mathrm{n}=15)\end{array}$ \\
\hline Age (yr) & $43.4 \pm 7.4$ \\
Total scar length (cm) & $8.3 \pm 1.9$ \\
Treatment scar length (cm) & $4.2 \pm 1.1$ \\
Initiation of laser treatment (day) & $14.0 \pm 1.7$ \\
Type of surgery & \\
$\quad$ Nipper sparing mastectomy+direct to implant & $12(80)$ \\
$\quad$ Skin sparing mastectomy+tissue expander insertion & $3(20)$ \\
Adjuvant therapy & \\
$\quad$ Chemotherapy & $3(20)$ \\
\hline
\end{tabular}

Values are presented as mean $\pm \mathrm{SD}$ or number (\%).

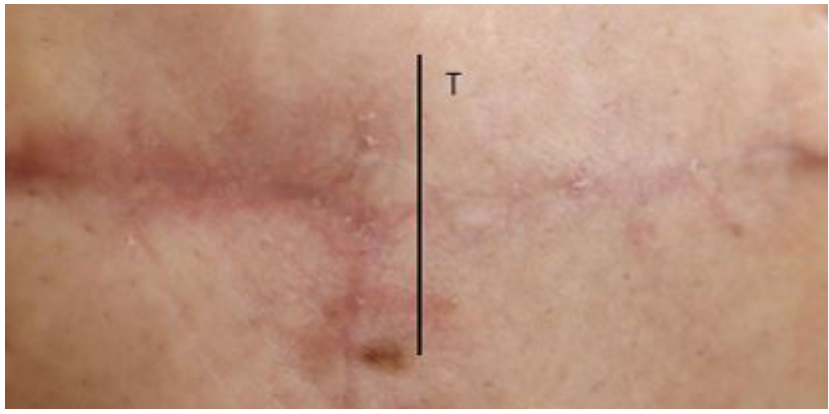

Fig. 2. Clinical photograph of patient No. 2. Six months after ablative $\mathrm{CO}_{2}$ fractional laser treatment of mastectomy scarring, the patient showed clinical improvement in the treated $(T)$ versus the untreated scar.

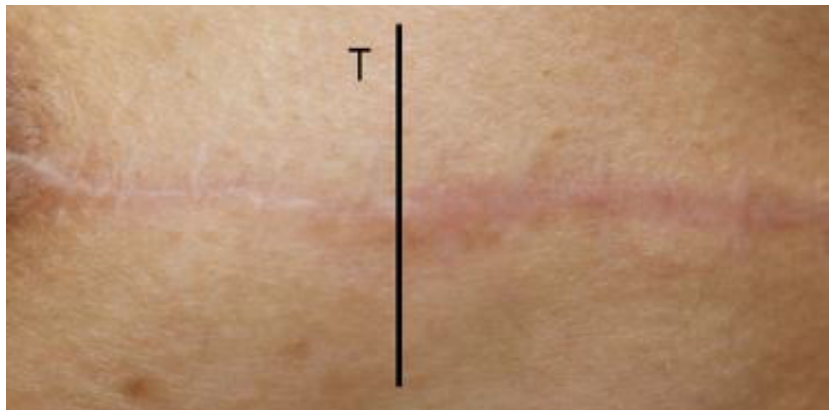

Fig. 3. Clinical photograph of patient No. 5. Six months after ablative $\mathrm{CO}_{2}$ fractional laser treatment of mastectomy scarring, the patient showed clinical improvement in the treated $(T)$ versus the untreated scar.
$2.96 \pm 1.40$ for the control side. There was a significant difference in the VSS score between the treatment side and the control side $(\mathrm{P}=0.002)$. The mean VAS scores were $4.13 \pm 1.36$ for the treatment side and $4.67 \pm 1.53$ for the control side. There was a significant difference in VAS scores between the treatment side and the control side $(\mathrm{P}=0.02)$ (Table 2, Fig. 4). Post-therapy crusting and transient erythema were reported, but all cases resolved in 1 week. No other adverse events, including posttherapy blister formation or infection, were observed.

The biopsy showed well-formed dermal scar lesions in the untreated and treated cases. ACFL treatment revealed increased reticular dermal collagen deposition with normal architecture, more organized collagen fibers, and a thickened epidermis with granular layer hyperplasia and normal stratum corneum (Fig. 5). Masson's trichrome staining revealed increased dermal collagen in the treatment group, and the collagen fibers were more regularly arranged (Fig. 6). These histologic findings are consistent with the clinical outcomes.

\section{DISCUSSION}

This was a randomized, comparative, prospective, split-scar study with blinding, in which we evaluated the effects of early ACFL treatment for mastectomy scars using VSS scores, VAS

Table 2. Mean VSS scores

\begin{tabular}{lccc}
\hline VSS parameter & Treatment & Control & P-value \\
\hline Vascularity & 0.44 & 0.59 & 0.002 \\
Pigmentation & 0.67 & 0.74 & 0.318 \\
Pliability & 0.96 & 0.74 & 0.006 \\
Height & 0.13 & 0.34 & 0.035 \\
Total & 2.20 & 1.29 & 0.002 \\
\hline
\end{tabular}

VSS, Vancouver scar scale.

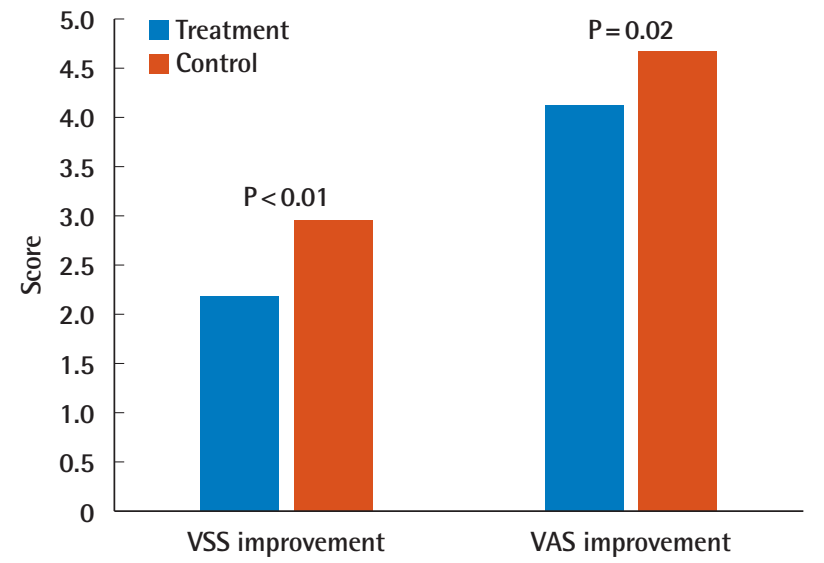

Fig. 4. Results of VSS and VAS scores. Statistically significant differences were observed in VSS and VAS scores. VSS, Vancouver scar scale; VAS, visual analog scale. 

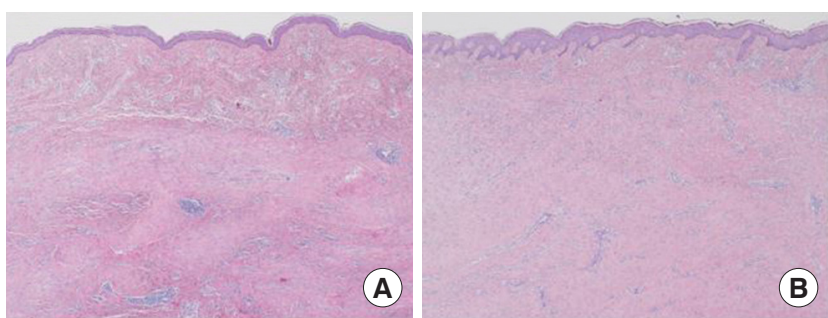

Fig. 5. Histological findings. Ablative $\mathrm{CO}_{2}$ fractional laser treatment revealed increased reticular dermal collagen deposition with normal architecture; more organized collagen fibers; and a thickened epidermis with granular layer hyperplasia and a normal stratum corneum $\left(H \& E_{1} \times 40\right)$. (A) Untreated and (B) treated.

scores, and histological findings. We found that scar texture was improved by ACFL treatment, and patients' VAS evaluations of scar appearance also showed an improvement in the treated scars compared to the untreated scars. Histopathological improvements were also observed on the treated side.

Postoperative scarring with physical sequelae (pain, itching) and the resulting psychological stress can affect not only patients' quality of life, but also their overall satisfaction with the surgical outcomes. These scars remind patients of their illness and may be associated with functional and psychosocial morbidity [10]. Although scar remodeling and clinical improvement require at least a few years after surgery, many patients hope that the scar will be improved as soon as possible.

Many expert opinions exist regarding which laser is ideal for select indications, but there is no consensus regarding the laser type and protocol for the treatment of surgical scars. Tierney et al. [11] showed that fractional photothermolysis was superior to a pulsed dye laser for the treatment of surgical scars. Park et al. [12] reported that ablative fractional laser treatment showed profound skin changes and collagen remodeling on rats when compared to nonablative fractional laser treatment. Kim et al. [13] showed that ablative fractional laser treatment for fresh thyroidectomy scars was more effective than nonablative fractional laser treatment. We therefore hypothesized that an ablative fractional laser would be more effective for surgical scars, and chose ACFL treatment rather than a nonablative fractional laser or a pulse dye laser.

Ablative fractional lasers, which were developed to compensate for the existing shortcomings of ablative surfacing lasers, are gaining popularity in scar treatment. The fractioning characteristics of these laser beams allow healthy skin to be maintained, accelerating the healing procedure [9]. Fractional lasers create a microthermal treatment zone (MTZ). These are repeated rows of heat damage that also penetrate the upper dermis. Each ablative zone is surrounded by healthy tissue. Spared, viable keratinocytes in healthy tissue can migrate to the MTZs, where they
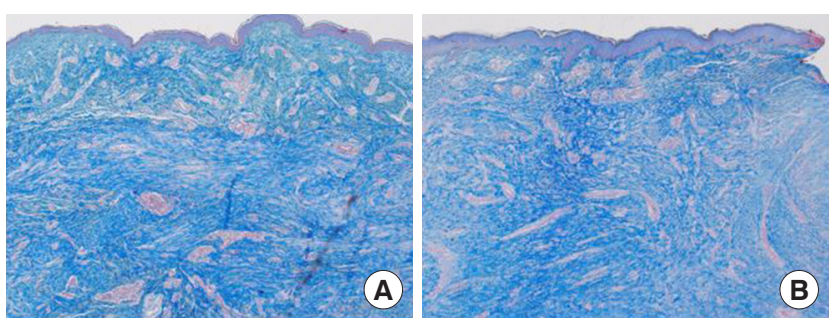

Fig. 6. Histological findings. Masson's trichrome staining $(\times 40)$ revealed poorly structured collagen fibers on the untreated side (A) and increased dermal collagen on the treated side (B).

promote the healing processes of reepithelization and collagen production [14]. Fractional lasers showed the same benefits as those obtained from ablative lasers, including dramatically reduced downtime and complications and straightforward resolution of common side effects such as erythema and edema [9]. We used pulse energy of 22-38 mJ with a total density of 300 spots $/ \mathrm{cm}^{2}$, and no patients developed any complications. We chose a relatively low energy level because the mastectomy skin flap was thin, and we considered the presence of the implant under the skin.

Modulation of the wound healing process may hold the key to minimize the formation of scarring. Wound healing may be divided into three overlapping phases: inflammation, proliferation, and remodeling. Traditionally, mature scars were the target of laser treatment. While laser treatment of mature scars has been shown to lead to some remodeling of scar tissue, early laser intervention for immature scars and even during wound healing has been proposed as a preventative approach to improve scar appearance. No consensus yet exists regarding the precise definition of an early intervention, but a recent systematic review defined it as laser treatment within 3 months after wound formation and found some improvement of scar appearance in $40 \%-75 \%$ of studies [7]. However, the proper window for laser treatment remains controversial. The current study evaluated the potential of ACFL treatment as an early intervention to improve the appearance of post-surgical scars. We treated patients with lasers within 2 to 3 weeks of surgery, at the time of suture removal, because we believed that re-epithelization would be complete at this point.

Previous reports have suggested that ACFL treatment has an effect on surgical scarring $[8,15,16]$. However, no studies have evaluated the therapeutic efficacy and safety of ACFL on mastectomy scars. To our knowledge, this is the first study to demonstrate clinical outcomes of ACFL treatment for mastectomy scars and safety after implant-based breast reconstruction in the early postoperative period. Furthermore, through a uniform comparison of scars, we able to prevent potential errors that 
may arise from differences in the scar location and suture method. However, this study also has some limitations. First, the number of samples of the patient was small. Second, while the scar formation takes at least a few years, the follow-up observations period in our study was shorter than the usual period. Third, although the histological results indicate positive effects of ACFL treatment, it should be kept in mind that the histological evaluations were done for part of the specimens, not for the whole specimens. Therefore, future studies should consider longer follow-up periods, larger sample sizes, and biopsies of all scars.

This study demonstrated that early scar treatment using ACFL showed a significant improvement in the clinical results of treatment compared to the untreated scars using VSS scores, VAS scores for patient satisfaction, and histological findings, and found that ACFL treatment had an impact on patient satisfaction. Therefore, early treatment of surgical scars with ACFL is recommended to achieve better scar cosmesis.

\section{NOTES}

\section{Conflict of interest}

Hyun Woo Shin is an editorial board member of the journal but was not involved in the peer reviewer selection, evaluation, or decision process of this article. No other potential conflicts of interest relevant to this article were reported.

\section{Ethical approval}

The study was approved by the Institutional Review Board of Kangbuk Samsung Hospital (IRB No. 2019-05-047) and performed in accordance with the principles of the Declaration of Helsinki. Written informed consent was obtained.

\section{Patient consent}

The patients provided written informed consent for the publication and the use of their images.

\section{Author contribution}

Conceptualization: HW Shin, KC Yoon. Data curation: S Suk, SW Chae. Project administration: HW Shin. Visualization: KC Yoon, J Kim. Writing - original draft: S Suk. Writing - review \& editing: S Suk. Approval of final manuscript: all authors.

\section{ORCID}

Hyun Woo Shin

Sangwoo Suk

Seoung Wan Chae

Kun Chul Yoon https://orcid.org/0000-0003-4396-3395

https://orcid.org/0000-0002-7647-841X https://orcid.org/0000-0003-0406-4469 https://orcid.org/0000-0001-9167-6716
Junekyu Kim

https://orcid.org/0000-0002-8904-5605

\section{REFERENCES}

1. Siegel RL, Miller KD, Jemal A. Cancer statistics, 2017. CA Cancer J Clin 2017;67:7-30.

2. Bray F, Ferlay J, Soerjomataram I, et al. Global cancer statistics 2018: GLOBOCAN estimates of incidence and mortality worldwide for 36 cancers in 185 countries. CA Cancer J Clin 2018;68:394-424.

3. Kang SY, Kim YS, Kim Z, et al. Basic findings regarding breast cancer in Korea in 2015: data from a breast cancer registry.J Breast Cancer 2018;21:1-10.

4. Sabino J, Lucas DJ, Shriver CD, et al. NSQIP analysis: increased immediate reconstruction in the treatment of breast cancer. Am Surg 2016;82:540-5.

5. Bayat A, McGrouther DA, Ferguson MW. Skin scarring. BMJ 2003;326:88-92.

6. Sobanko JF, Vachiramon V, Rattanaumpawan P, et al. Early postoperative single treatment ablative fractional lasing of Mohs micrographic surgery facial scars: a split-scar, evaluator-blinded study. Lasers Surg Med 2015;47:1-5.

7. Karmisholt KE, Haerskjold A, Karlsmark T, et al. Early laser intervention to reduce scar formation: a systematic review. J Eur Acad Dermatol Venereol 2018;32:1099-110.

8. Jang JU, Kim SY, Yoon ES, et al. Comparison of the effectiveness of ablative and non-ablative fractional laser treatments for early stage thyroidectomy scars. Arch Plast Surg 2016;43:575-81.

9. Manstein D, Herron GS, Sink RK, et al. Fractional photothermolysis: a new concept for cutaneous remodeling using microscopic patterns of thermal injury. Lasers Surg Med 2004;34:426-38.

10. Brown BC, McKenna SP, Siddhi K, et al. The hidden cost of skin scars: quality of life after skin scarring. J Plast Reconstr Aesthet Surg 2008;61:1049-58.

11. Tierney E, Mahmoud BH, Srivastava D, et al. Treatment of surgical scars with nonablative fractional laser versus pulsed dye laser: a randomized controlled trial. Dermatol Surg 2009;35:1172-80.

12. Park SH, Kim DW, Jeong T. Skin-tightening effect of fractional lasers: comparison of non-ablative and ablative fractional lasers in animal models. J Plast Reconstr Aesthet Surg 2012;65:1305-11.

13. Kim HS, Lee JH, Park YM, et al. Comparison of the effectiveness of nonablative fractional laser versus ablative fractional laser in thyroidectomy scar prevention: a pilot study. J Cosmet Laser Ther 2012;14:89-93. 
14. Magnani LR, Schweiger ES. Fractional CO2 lasers for the treatment of atrophic acne scars: a review of the literature. J Cosmet Laser Ther 2014;16:48-56.

15. Safra T, Shehadeh W, Koren A, et al. Early intervention with pulse dye and $\mathrm{CO} 2$ ablative fractional lasers to improve cutaneous scarring post-lumpectomy: a randomized con- trolled trial on the impact of intervention on final cosmesis. Lasers Med Sci 2019;34:1881-7.

16. Karmisholt KE, Taudorf EH, Wulff CB, et al. Fractional $\mathrm{CO} 2$ laser treatment of caesarean section scars: a randomized controlled split-scar trial with long term follow-up assessment. Lasers Surg Med 2017;49:189-97. 